\title{
Assessment of left atrial functional parameters using a novel dedicated analysis tool for real-time three-dimensional echocardiography: validation in comparison to magnetic resonance imaging
}

\author{
Ronny R. Buechel • Gregor Sommer • Gregor Leibundgut • Andreas Rohner • \\ Florian Riede $\cdot$ Arnheid Kessel-Schaefer • Beat A. Kaufmann - Michael J. Zellweger • \\ Jens Bremerich • Michael Handke
}

Received: 15 May 2012/Accepted: 11 September 2012/Published online: 23 September 2012

(C) Springer Science+Business Media Dordrecht 2012

\begin{abstract}
Real-time three-dimensional echocardiography (RT3DE) is superior to two-dimensional echocardiography in assessing left atrial (LA) parameters, but to date algorithms developed for the left ventricle were applied due to a lack of dedicated LA software. In addition, no data are available on RT3DE assessment of active atrial contraction. The aim of this study was to validate a novel RT3DE analysis tool specifically dedicated to evaluate the LA. Cardiac magnetic resonance imaging (MRI) served as standard of reference. Fifty-five patients scheduled for pulmonary vein isolation underwent cardiac MRI and RT3DE. On ultrasound image datasets, a dynamic polyhedron model of the LA was generated from which LA maximum and minimum volumes $\left(\mathrm{LA}_{\max }\right.$ and $\mathrm{LA}_{\min }$ ), passive atrial emptying fraction (LAEF), and active atrial ejection fraction $\left(\mathrm{LAEF}_{\text {true }}\right)$ were derived and compared to values obtained from cardiac MRI. High intraclass correlations between RT3DE and MRI were found for $\mathrm{LA}_{\max }$
\end{abstract}

Electronic supplementary material The online version of this article (doi:10.1007/s10554-012-0127-0) contains supplementary material, which is available to authorized users.

R. R. Buechel $(\bowtie) \cdot$ G. Leibundgut · A. Rohner · F. Riede

A. Kessel-Schaefer · B. A. Kaufmann - M. J. Zellweger ·

M. Handke

Department of Cardiology, University Hospital Basel,

Petersgraben 4, 4031 Basel, Switzerland

e-mail: ronbuechel@tiscali.ch; buechelr@uhbs.ch

G. Sommer · J. Bremerich

Department of Radiology, University Hospital Basel,

Petersgraben 4, 4031 Basel, Switzerland

M. Handke

Division of Cardiology, Basel University Medical Clinic,

Rheinstrasse 26, 4410 Liestal, Switzerland $(\mathrm{r}=0.94, p<0.001), \mathrm{LA}_{\min }(\mathrm{r}=0.95, p<0.001), \mathrm{LAEF}$ ( $\mathrm{r}=0.92, p<0.001)$, and $\mathrm{LAEF}_{\text {true }}(\mathrm{r}=0.87, p<0.001)$. Similarly, Bland-Altman analysis revealed narrow limits of agreement for $\mathrm{LA}_{\max }$ (-28.6 to $\left.14.1 \mathrm{ml}\right), \mathrm{LA}_{\min }(-26.8$ to $12.4 \mathrm{ml}), \mathrm{LAEF}$ ( -11.2 to $14.9 \%)$, and $\mathrm{LAEF}_{\text {true }}(-10.6$ to $6.8 \%)$. $\mathrm{LA}_{\max }, \mathrm{LA}_{\min }$ and $\mathrm{LAEF}_{\text {true }}$ were measured significantly $(p<0.05)$ lower by RT3DE $(111 \pm 38 \mathrm{ml}$ vs. $118 \pm 39 \mathrm{ml}, 73 \pm 38 \mathrm{ml}$ vs. $80 \pm 41 \mathrm{ml}$, and $23 \pm 14 \%$ vs. $27 \pm 14 \%$, respectively). Interobserver and intraobserver RT3DE measurements correlated closely. RT3DE using a novel dedicated software tool is valid, accurate and reproducible for assessing LA dimensional and functional parameters. This study corroborates previous reports and extends its validity to the assessment of active LA contraction.

Keywords Echocardiography · Real-time three-dimensional echocardiography · Left atrial systolic function - Left atrial active contraction

\section{Introduction}

There is growing evidence that left atrial (LA) size and function serves as an important diagnostic and prognostic factor in a variety of conditions [1-7]. Furthermore, recent advances in pulmonary vein isolation (PVI) for the treatment of atrial fibrillation have further increased the interest in accurately imaging LA function [8]. While magnetic resonance imaging (MRI) represents the current gold standard for assessment of LA size and function [9], the technique is not very well suited for routine or even serial assessment of the LA due to a lack of portability and availability combined with the relatively high cost of this modality. 
By contrast, echocardiography is inexpensive, widely available and portable and therefore preferable to evaluate LA dimensions and functional parameters. However, as of today the echocardiographic assessment of LA size and function relied heavily on estimates based on M-mode and two-dimensional (2D) echocardiography. While M-mode echocardiography has traditionally been used to make assumptions on LA size by measuring the LA diameter, calculation of LA volumes using the arealength-method, the ellipsoid formula or biplane methods were shown to be superior in terms of accuracy and prognostic value [10]. Both methods, however, rely on geometric assumptions and may therefore reflect relatively rough approximations.

The recent advent of real-time three-dimensional echocardiography (RT3DE) has enabled cardiologists to obtain LA volumes and functional parameters based on real anatomical conditions and has been shown to be superior to conventional two-dimensional 2D techniques [11-14]. Previously published studies assessing LA functional parameters using RT3DE, however, had to rely on semiautomatic contour tracing algorithms originally developed for the left ventricle [15-17].

A novel software analysis tool specifically dedicated to the functional assessment of the LA has recently been introduced and allows obtaining comprehensive global as well as regional LA functional parameters [18]. Moreover, beside calculation of the passive atrial emptying fraction (LAEF) it also enables to assess functional parameters concerning the active contraction of the LA during each heart cycle $\left(\mathrm{LAEF}_{\text {true }}\right)$ and potentially even LA dyssynchrony parameters.

The aim of the present study was to clinically validate this novel RT3DE analysis tool with cardiac MRI serving as the standard of reference.

\section{Methods}

\section{Patient population}

Sixty consecutive patients scheduled for pulmonary vein isolation (PVI) due to symptomatic persistent or paroxysmal atrial fibrillation who underwent clinically indicated cardiac MRI were prospectively enrolled in the present study. RT3DE was performed in all patients on the same day as MRI and patients were enrolled regardless of the quality of the acoustic window obtained during echocardiography.

Informed consent was obtained from all patients and the study protocol was approved by the local institutional review board.
RT3DE image acquisition and post-processing

RT3DE datasets were acquired using a Philips IE33 ${ }^{\mathrm{TM}}$ ultrasound system equipped with a matrix array transducer (X3-1 and X5-1 transducers, Philips Medical Systems, Andover, USA). An apical view enabling full coverage of the LA was selected and lateral sector size was carefully adjusted to achieve the highest possible frame rate during image acquisition. Trigger delay was set to $300 \mathrm{~ms}$ after the electrocardiographic QRS complex to ensure temporal coverage of the entire diastole. On average, two to four datasets were acquired per patient, from which the best dataset in terms of image quality, frame rate and correct electrocardiographic triggering was selected.

All RT3DE datasets were transferred to a dedicated workstation for final offline analysis of left atrial volumes and functional parameters using a novel software tool (4D LA Analysis $^{\odot}$, TomTec Imaging Systems Inc., Unterschleissheim, Germany). Analysis was performed by an experienced cardiologist. Only a single dataset with the highest subjective overall quality was selected and used for analysis.

Details on the workflow of the LA analysis software have been previously published [18] and are demonstrated in the animation (Online Resource 1). In short, the reader uses mitral valve closure and mitral valve opening to manually define an end-diastolic (mitral valve opening) and an endsystolic frame (frame before mitral valve closure). For the definition of the start of active atrial contraction the frame immediately before a slight countermovement or re-opening of the mitral valve due to atrial systolic flow becomes visible during diastole was selected. In a further step, initial contours of the LA at end-diastole and end-systole were manually defined for the apical four-chamber, two-chamber, and longaxis view. Using an automated border-detection technique, a polyhedron model of the LA was automatically created which was manually corrected. Pulmonary vein orifices and/ or left atrial appendage were not included into the contour. Finally, maximum and minimum LA volumes $\left(\mathrm{LA}_{\max }\right.$ and $\mathrm{LA}_{\text {min }}$ ), left atrial emptying fraction (LAEF, encompassing the fraction ejected through both passive suction and active atrial contraction), and left atrial active ejection fraction $\left(\mathrm{LAEF}_{\text {true }}\right.$, describing only of the ejection fraction generated by active atrial contraction) were calculated for analysis (Fig. 1).

MRI image acquisition and post-processing

MRI studies were performed using a $1.5 \mathrm{~T}$ clinical MRI system (Magnetom Espree, Siemens Medical Solutions, Erlangen Germany) with multi-channel phased-array receiver coils (Total imaging matrix (TIM), Siemens Medical Solutions). Imaging was performed with the patient in a supine position. Localizing scans were followed by a series 


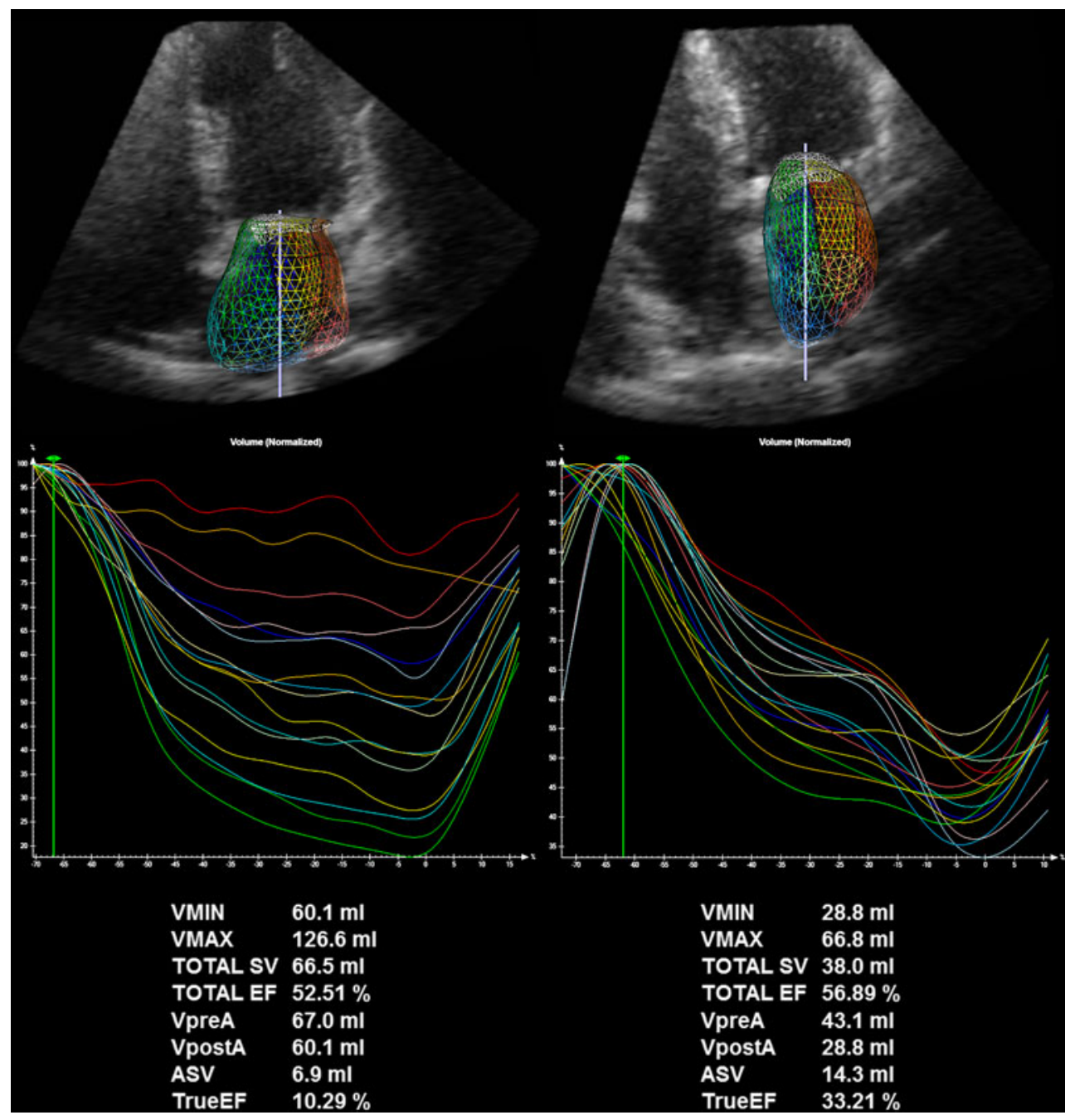

Fig. 1 Results of LA analysis of two patients diagnosed with paroxysmal atrial fibrillation who presented with sinus rhythm during image acquisition. On the left a severely enlarged LA (LA $A_{\max } 80 \mathrm{ml} /$ $\mathrm{m}^{2}$ ) and a markedly reduced $\mathrm{LAEF}_{\text {true }}$ is shown while the patient on the right presents with a slightly enlarged $\mathrm{LA}\left(\mathrm{LA}_{\max } 31 \mathrm{ml} / \mathrm{m}^{2}\right)$ and a substantially higher $\mathrm{LAEF}_{\text {true }} . \quad \mathrm{LA}=$ left atrium; $\quad \mathrm{VMIN}=$ $\mathrm{LA}_{\min }=$ minimum LA volume at end-diastole; $\mathrm{VMAX}=\mathrm{LA}_{\max }=$ maximum LA volume at end-systole; TOTAL SV = atrial stroke

of transversely oriented cine acquisitions using a balanced steady state free precession (b-SSFP) sequence (TR = $40 \mathrm{~ms}$, TE $=1.2 \mathrm{~ms}$, flip angle $63^{\circ}$, matrix $192 \times 156$, in plane resolution $1.6 \times 1.6 \mathrm{~mm}^{2}$ ). Slices were acquired to cover the LA with a section thickness of $6 \mathrm{~mm}$ and no interslice gaps. Each slice was obtained during breath-hold at expiration with retrospective electrocardiography gating. Temporal resolution was 25 frames per heart cycle using parallel imaging with an acceleration factor of 2 .

All MRI datasets were transferred to a dedicated workstation and were analyzed by an experienced radiologist blinded to the results of 3DRTE. A dedicated software tool was used (Argus, Siemens Medical Solutions) and the LA border was contoured in axial slices, while the volume throughout the entire diastole (passive emptying and atrial contraction); TOTAL EF $=\mathrm{LAEF}=\mathrm{LA}$ emptying fraction regarding the entire diastole $(\mathrm{Vmax}-\mathrm{Vmin} / \mathrm{Vmax}) ; \mathrm{VpreA}=\mathrm{LA}$ volume at the beginning of atrial contraction; Vpost $\mathrm{A}=\mathrm{LA}$ volume at the end of atrial contraction; $\mathrm{ASV}=$ stroke volume during atrial contraction $\left(\right.$ VpreA-VpostA); TrueEF $=\mathrm{LAEF}_{\text {true }}=\mathrm{EF}$ during atrial contraction (VpreA-VpostA/VpreA)

timing of LA volume measurements and calculation of functional parameters were identical to that described under 3DRTE acquisition methods. As with RT3DE, pulmonary vein orifices and/or left atrial appendage were not included into the contour.

\section{Interobserver and intraobserver agreement}

Interobserver agreement was assessed from 10 randomly selected images by a second reader blinded to the results of the first reader and those obtained by MRI. Intraobserver agreement was assessed by repeated measurements from 10 randomly selected images by the same observer at least 1 month after the first analysis. For each subject, the same 
RT3DE dataset used for the initial measurements by the first reader were used by the second reader [16, 17].

Statistics

SPSS 18 (SPSS Inc.) was used for statistical testing. Quantitative variables were expressed as mean \pm standard deviation and categorical variables as frequencies or percentages. Paired $t$ test and Wilcoxon rank-sum test were used for parametric and non-parametric variables, respectively, to test for differences between parameters acquired during scans. The correlation of dimensional and functional parameters between RT3DE and MRI as well as interobserver and intraobserver agreement was assessed by calculating Bland-Altman limits of agreement and coefficients of variation $(\mathrm{CV})$. In addition, Bland-Altman limits of agreement were calculated. $p$ values of less than 0.05 were considered statistically significant and the $95 \%$ confidence intervals are presented where suitable.

\section{Results}

Patient population characteristics

Out of 60 enrolled patients, image acquisition did not yield usable data for conclusive LA analysis in 5 patients (9\%), i.e. 2 RT3DE datasets with insufficient frame rates and 3 MRI datasets of insufficient image quality. Thus, final analysis and comparison to MRI was performed in 55 patients. Patient baseline characteristics are given in Table 1.

Image acquisition

Mean frame rate during RT3DE acquisition was $26 \pm 4 \mathrm{~Hz}$ (range 16-37 Hz). Heart rates during acquisition did not differ significantly between RT3DE (68 \pm 16 bpm, range 42-110 bpm) and MRI (64 $\pm 13 \mathrm{bpm}$, range 40-97 bpm).

During acquisition of RT3DE datasets 12 patients (22\%) presented with atrial fibrillation, while during MRI image acquisition 18 patients $(33 \%)$ had atrial fibrillation. Out of 55 patients, 36 (65\%) showed sinus rhythm during both acquisitions, thus enabling to additionally assess active contraction parameters (i.e. calculating $\mathrm{LAEF}_{\text {true }}$ by defining the "preA"-frame). $\mathrm{LA}_{\max }, \mathrm{LA}_{\min }$ and LAEF were calculated in all 55 patients, as sinus rhythm is not mandatory for the calculation of these parameters.

Indexed LA volumes as assessed by RT3DE and MRI are given in Table 2. Of note, when indexed to body surface as recommended by guidelines [19] a majority of the subjects $(\mathrm{n}=45,82 \%)$ included in this study presented with $\mathrm{LA}_{\max }$ above the reference values for RT3DE as suggested by Aune et al. [20].
Table 1 Patient baseline characteristics $(n=55)$

\begin{tabular}{ll}
\hline $\begin{array}{l}\text { Male } \\
\text { Age (years) }\end{array}$ & $37(67 \%)$ \\
Mean \pm standard deviation & $61 \pm 10$ \\
Range & $32-77$ \\
Body mass index $\left(\mathrm{kg} / \mathrm{m}^{2}\right)$ & \\
Mean \pm standard deviation & $27 \pm 5$ \\
Range & $19-40$ \\
Left ventricular ejection fraction $(\%)$ & \\
Mean \pm standard deviation & $55 \pm 8$ \\
Range & $35-75$ \\
Cardiovascular risk factors & \\
Obesity (BMI $\left.>30 \mathrm{~kg} / \mathrm{m}^{2}\right)$ & $13(24 \%)$ \\
Smoking & $7(13 \%)$ \\
Diabetes mellitus & $3(5 \%)$ \\
Hypertension & $32(58 \%)$ \\
Dyslipidaemia & $24(44 \%)$ \\
Positive family history & $17(31 \%)$ \\
Type of atrial fibrillation & \\
Paroxysmal & $38(69 \%)$ \\
Persistent & $17(31 \%)$ \\
\hline
\end{tabular}

Table 2 Indexed left atrial volumes assessed by MRI and RT3DE $(\mathrm{n}=55)$

\begin{tabular}{lll}
\hline & $\begin{array}{l}\text { MRI } \\
\text { Mean } \pm \text { SD (range) }\end{array}$ & $\begin{array}{l}\text { RT3DE } \\
\text { Mean } \pm \text { SD (range) }\end{array}$ \\
\hline $\mathrm{LA}_{\max }\left(\mathrm{ml} / \mathrm{m}^{2}\right)$ & $60 \pm 17(30-106)$ & $56 \pm 16(27-106)$ \\
$\mathrm{LA}_{\min }\left(\mathrm{ml} / \mathrm{m}^{2}\right)$ & $40 \pm 20(13-102)$ & $37 \pm 18(13-101)$ \\
\hline
\end{tabular}

$S D$ standard deviation, MRI magnetic resonance imaging, RT3DE real-time three-dimensional echocardiography, $L A_{\max }$ maximum LA volume at end-systole, $L A_{\min }$ minimum LA volume at end-diastole

\section{Comparison of RT3DE versus MRI}

LA volumes were significantly lower when assessed by RT3DE than by MRI $\left(\right.$ LA $_{\max } 111 \pm 38 \mathrm{ml}$ vs. $118 \pm 39 \mathrm{ml}$, $p<0.001 ; \mathrm{LA}_{\min } 73 \pm 38 \mathrm{ml}$ vs. $\left.80 \pm 41 \mathrm{ml}, p<0.001\right)$, while LAEF showed a trend towards slightly higher percentages when assessed by RT3DE (36 $\pm 17 \%$ vs. $34 \pm 18 \%, p=0.051)$. Regarding the active component of atrial contraction, $\mathrm{LAEF}_{\text {true }}$ was significantly lower when assessed by RT3DE in comparison to MRI ( $23 \pm 14 \%$ vs. $27 \pm 14 \% ; p<0.05$ ) (Fig. 2).

All functional parameters assessed by RT3DE correlated highly with MRI as shown in Fig. 3 with intraclass correlation coefficients ranging from 0.87 to 0.95 .

In addition, Bland-Altman analysis (Fig. 4) revealed narrow limits of agreement for $\mathrm{LA}_{\max }(-28.6$ to $14.1 \mathrm{ml})$, $\mathrm{LA}_{\min }$ ( -26.8 to $12.4 \mathrm{ml}$ ), LAEF ( -11.2 to $14.9 \%$ ), and $\mathrm{LAEF}_{\text {true }}(-10.6$ to $6.8 \%)$. 


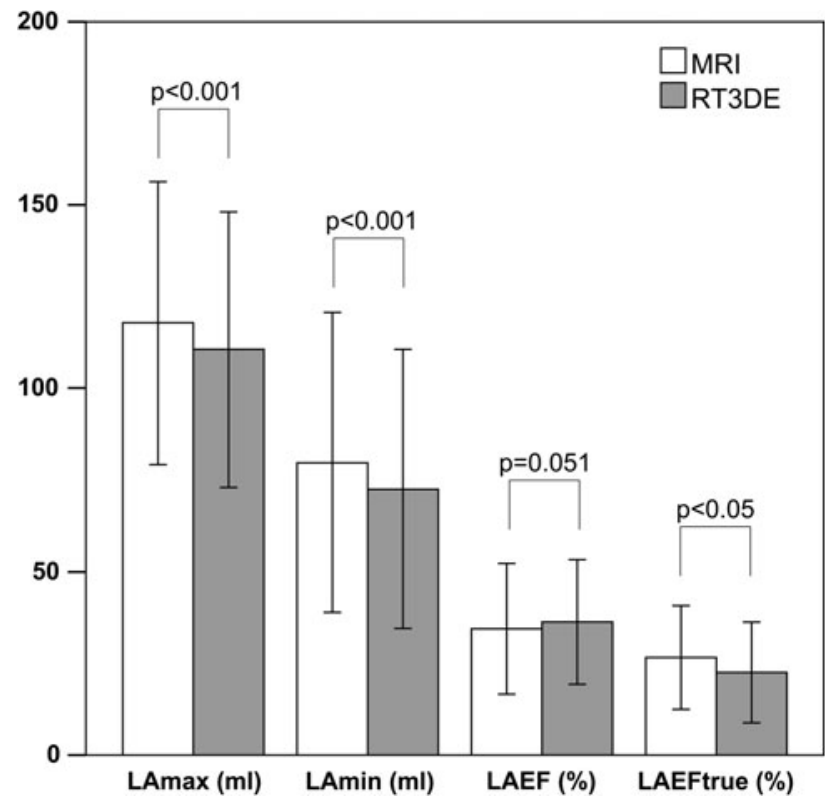

Fig. 2 Mean values of LA dimensional and functional parameters obtained by MRI in comparison to RT3DE. Error bars indicate the standard deviation. Maximum $\left(\mathrm{LA}_{\max }\right)$ and minimum $\left(\mathrm{LA}_{\min }\right) \mathrm{LA}$ volumes as well as the active LA ejection fraction $\left(\mathrm{LAEF}_{\text {true }}\right)$ were significantly lower when assessed by RT3DE than by MRI, whereas the passive emptying fraction (LAEF) showed a trend towards slightly higher values

\section{Reproducibility}

Interobserver agreement as assessed by Bland-Altman analysis and calculation of coefficients of variation revealed narrow limits of agreement and similar variance for both observers for $\mathrm{LA}_{\max }$ ( -7.0 to $14.7 \mathrm{ml}$; CV $0.28 \mathrm{vs}$. $0.25), \mathrm{LA}_{\min }(-9.4$ to $14.6 \mathrm{ml}$; $\mathrm{CV} 0.27$ vs. 0.25), LAEF ( -8.1 to $10.2 \%$; CV 0.23 vs. 0.25$)$, and $\mathrm{LAEF}_{\text {true }}(-7.2$ to $8.9 \%$; CV 0.69 vs. 0.70). Intraobserver agreement analysis showed similarly narrow limits of agreement and variance for $\mathrm{LA}_{\max }$ (-8.4 to $7.4 \mathrm{ml}$; CV 0.22 vs. 0.22), $\mathrm{LA}_{\min }(-7.0$ to $8.8 \mathrm{ml}$; $\mathrm{CV} 0.24$ vs. 0.26 ), $\mathrm{LAEF}$ ( -7.2 to $7.2 \%$; $\mathrm{CV}$ 0.32 vs. 0.34$)$, and $\mathrm{LAEF}_{\text {true }}(-3.8$ to $2.7 \%$; CV 0.62 vs. $0.55)$.

\section{Discussion}

The present study demonstrates that RT3DE using a dedicated analysis tool is a valid and highly accurate method for measuring passive and active LA functional parameters. Our data demonstrate very high correlations for $\mathrm{LA}_{\max }$, $\mathrm{LA}_{\text {min }}$, LAEF, and $\mathrm{LAEF}_{\text {true }}$ when compared to cardiac MRI. In line with previous studies comparing RT3DE assessment of the LA to MRI or CT, the present data demonstrate a consistent underestimation of RT3DE volumes [15, 18].
In the present study, however, the differences were less pronounced than previously reported [15, 18]. The higher spatial resolution of MRI in comparison to RT3DE has been repeatedly suggested as a likely explanation for the general underestimation of LA volumes by RT3DE along with a possible inclusion of pulmonary venous confluence or even part of the LA appendage into the actual LA volume when assessed by MRI. Of note, in the present study, we have used axial slices for LA volume assessment by MRI which enables better delineation of the pulmonary vein orifices, thus eliminating accidental inclusion of the venous confluence into the LA volume [21]. By contrast, using axial slices instead of a short axis stack may itself have an impact on volume calculation, possibly leading to higher volumes by MRI. Furthermore, in 2D and 3D echocardiography, the apical four-chamber view places the left atrium at the far field of the ultrasound beam, resulting in loss of lateral image resolution. In the present study, however, an apical view with full coverage of the centered LA was selected and lateral sector size was carefully adjusted to achieve the highest possible frame rate during image acquisition potentially leading to improved overall image quality in terms of better atrial wall delineation and/ or higher frame rates during acquisition. This may have contributed to the less pronounced differences in LA volumes in the present study. Although the use of different standard references, namely MRI and CT, in previous studies may to some extent have further contributed to a less pronounced underestimation of RT3DE volumes in the present study, we cannot further comment on this as it was beyond the scope of the present study to assess differences in LA volume assessment between MRI and CT. Importantly, Bland-Altman limits of agreement were narrow and comparable to previously published results [18]. Nevertheless, while our data suggest that functional parameters of the LA can very accurately be assessed by RT3DE, it has to be kept in mind that the systematic underestimation of volumes by RT3DE constitutes a difference that may have clinical significance.

In the present study, only $2(4 \%)$ patients had to be excluded from LA analysis due to non-evaluable RT3DE datasets, both owing to insufficient frame rates. In fact, no subjects were excluded from RT3DE analysis due to insufficient image quality. The latter, along with a high interobserver and intraobserver agreement, further underlines the robustness of this technique and its suitability for clinical use in an every-day setting.

While accuracy and consistency for LA volumes and the passive emptying fraction have been shown in a previous study comparing the same dedicated LA RT3DE analysis tool with CT [18], no data have been available on the accuracy of left atrial active contraction (i.e. $\mathrm{LAEF}_{\text {true }}$ ). In fact, to the best of our knowledge, the present study is the 

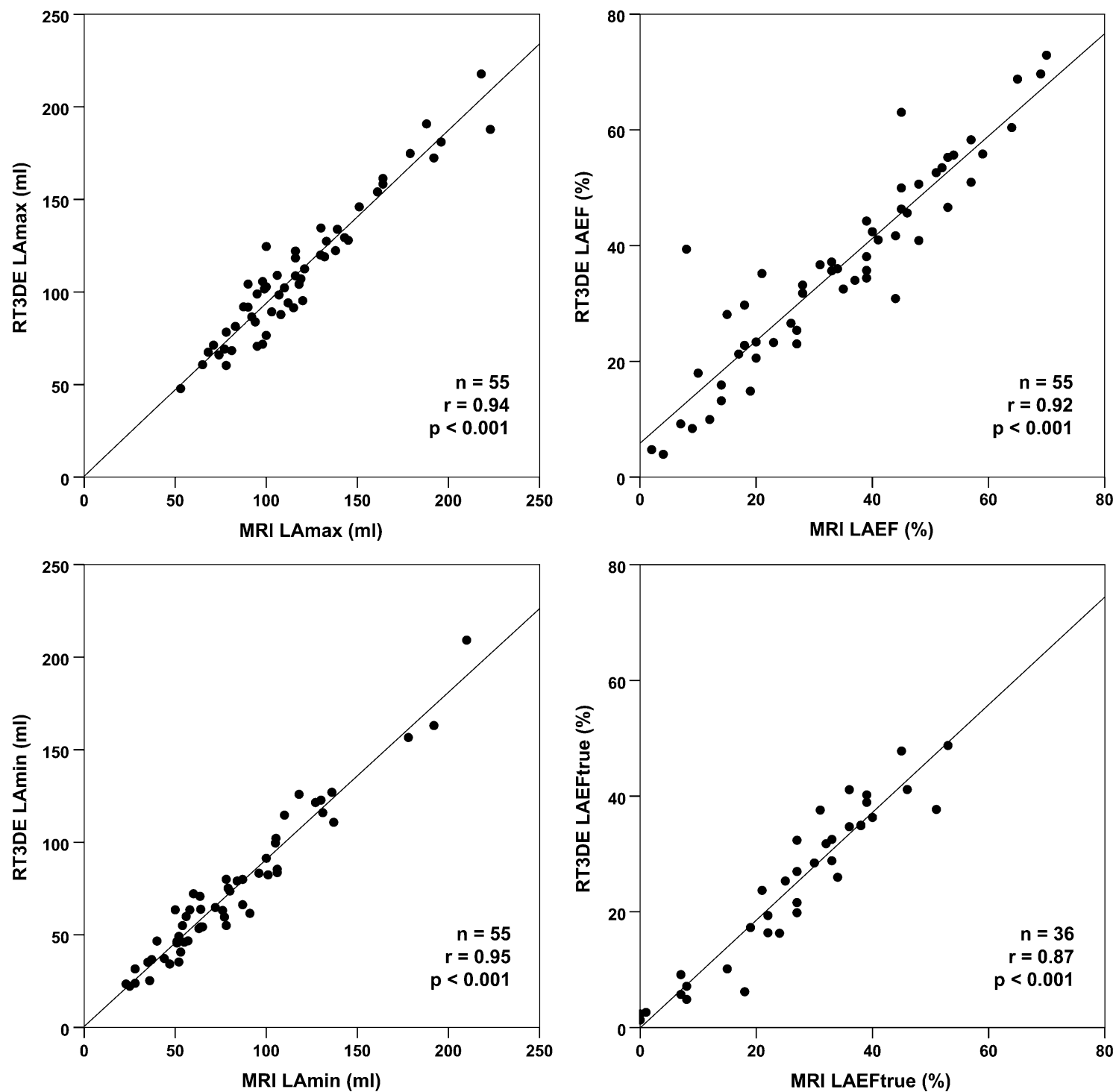

Fig. 3 Linear regression analysis comparing measurements obtained by RT3DE to MRI. $L A_{\max }$ maximum LA volume at end-systole, $L A_{\min }$ minimum LA volume at end-diastole, $L A E F$ LA emptying fraction, $L A E F_{\text {true }}$ LA ejection fraction during atrial contraction

first to validate echocardiographic LA active contraction measurements using a dedicated tool for the LA against an independent reference technique. This is of importance, as recent advances in PVI for the treatment of atrial fibrillation and the increasing application of cardiac resynchronization therapy for heart failure may prospectively even further augment the interest in an accurate assessment particularly of the active component of LA function [22, 23]. Against this background, a dedicated analysis tool for RT3DE that allows in-depth analysis of the LA as presented in our study has the potential to further expand our knowledge and understanding in this field of research where an easily applicable, comprehensive and economical diagnostic tool is highly welcome. RT3DE potentially even allows assessing LA synchrony and dyssynchrony parameters. Further studies assessing the latter as well as the prognostic value of overall and regional LA functional parameters in various conditions, however, are needed to fully reveal the clinical potential of LA assessment using RT3DE.

\section{Limitations}

It may be perceived as a limitation that the present study evaluated patients of a cohort referred to PVI rather than a normal population. Thus, a majority of patients $(n=45$, $82 \%$ ) expectedly presented with a dilated LA. This, however, allowed us to cover a broader range of volumes. The high overall correlation and consistently narrow Bland-Altman limits of agreement even for dilated atriae 

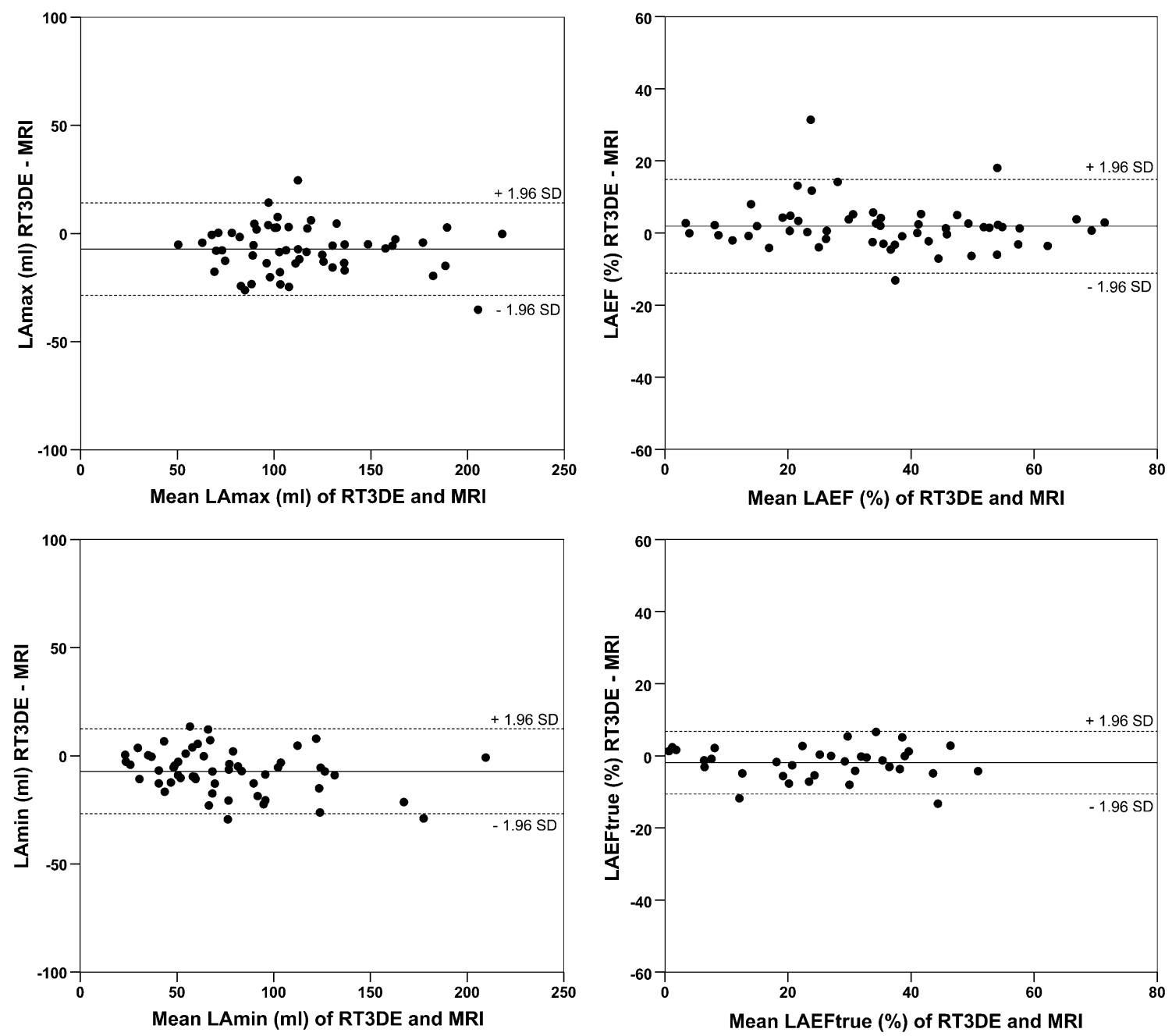

Fig. 4 Bland-Altman plots comparing measurements obtained by RT3DE and MRI. $L A_{\max }$ maximum LA volume at end-systole, $L A_{\text {min }}$ minimum LA volume at end-diastole, $L A E F$ LA emptying fraction, $L A E F_{\text {true }}$ LA ejection fraction during atrial contraction

further emphasize the applicability of the LA analysis tool in this clinically relevant population. We did not compare the dedicated LA analysis tool to other echocardiographic methods previously used for the assessment of LA functional parameters as the aim of the present study was particularly to validate this novel technique against the gold standard MRI.

In addition to the accuracy of the measurement of volumetric datasets, differences in the timing of events during the cardiac cycle could also influence the values obtained. While this can be assumed to be less of an issue for maximal and minimal volumes, which are derived from the volumetric dataset independent of the definition of endsystole and end-diastole, the calculation of $\mathrm{LAEF}_{\text {true }}$ depends on the definition of the onset of atrial contraction (i.e. the preA-frame). The pre-A frame was set carefully for both RT3DE and MRI datasets using the timing of mitral valve re-opening after diastolic diastasis. However, given the slightly different heart rates and imaging frame rates during RT3DE versus MRI, we are not able to assess whether there were small differences in the definition of the onset of active atrial contraction.

Finally, it may be perceived as a limitation that the software tools used for MRI and RT3DE analysis were not identical which may have led to systematic differences between both modalities.

\section{Conclusion}

RT3DE using a novel dedicated software tool is a valid, highly accurate and reproducible method to rapidly assess LA dimensional and functional parameters. The present study corroborates previous reports and extends its validity to the assessment of the active contraction of the LA. 
Acknowledgments The software package (4D LA Analysis ${ }^{\odot}$ ) for RT3DE analysis used in this study was provided free of charge by TomTec Imaging Systems, Unterschleissheim, Germany.

Disclosures We wish to acknowledge that the patient population analysed in the present study is shared with another scientific work currently under peer-review with a different journal. In contrast to the present study which aims at validation particularly of the active component of atrial ejection, the second study compares various twodimensional and three-dimensional echocardiographic methods for LA volume analysis (but not $\mathrm{LAEF}_{\text {true }}$ ) with MRI serving as standard of reference. Of note, the same 3DRTE and MRI acquisitions used in the present study were also included into the other study. However, offline-analysis of raw datasets was independently performed for both works. Thus, while certain methodological aspects are identical for both, the studies' backgrounds, results, scope and conclusions differ substantially.

\section{Conflict of interest None.}

\section{References}

1. Benjamin EJ, D'Agostino RB, Belanger AJ, Wolf PA, Levy D (1995) Left atrial size and the risk of stroke and death. The Framingham Heart Study. Circulation 92(4):835-841

2. Matsuda M, Matsuda Y (1996) Mechanism of left atrial enlargement related to ventricular diastolic impairment in hypertension. Clin Cardiol 19(12):954-959

3. Tsang TS, Barnes ME, Gersh BJ, Bailey KR, Seward JB (2002) Left atrial volume as a morphophysiologic expression of left ventricular diastolic dysfunction and relation to cardiovascular risk burden. Am J Cardiol 90(12):1284-1289

4. Moller JE, Hillis GS, Oh JK, Seward JB, Reeder GS, Wright RS, Park SW, Bailey KR, Pellikka PA (2003) Left atrial volume: a powerful predictor of survival after acute myocardial infarction. Circulation 107(17):2207-2212

5. Pritchett AM, Jacobsen SJ, Mahoney DW, Rodeheffer RJ, Bailey KR, Redfield MM (2003) Left atrial volume as an index of left atrial size: a population-based study. J Am Coll Cardiol 41(6): 1036-1043

6. Osranek M, Fatema K, Qaddoura F, Al-Saileek A, Barnes ME, Bailey KR, Gersh BJ, Tsang TS, Zehr KJ, Seward JB (2006) Left atrial volume predicts the risk of atrial fibrillation after cardiac surgery: a prospective study. J Am Coll Cardiol 48(4):779-786

7. Suh IW, Song JM, Lee EY, Kang SH, Kim MJ, Kim JJ, Kang DH, Song JK (2008) Left atrial volume measured by real-time 3-dimensional echocardiography predicts clinical outcomes in patients with severe left ventricular dysfunction and in sinus rhythm. J Am Soc Echocardiogr 21(5):439-445

8. To AC, Flamm SD, Marwick TH, Klein AL (2011) Clinical utility of multimodality LA imaging: assessment of size, function, and structure. JACC Cardiovasc Imaging 4(7):788-798

9. Jarvinen V, Kupari M, Hekali P, Poutanen VP (1994) Assessment of left atrial volumes and phasic function using cine magnetic resonance imaging in normal subjects. Am J Cardiol 73(15): $1135-1138$

10. Tsang TS, Abhayaratna WP, Barnes ME, Miyasaka Y, Gersh BJ, Bailey KR, Cha SS, Seward JB (2006) Prediction of cardiovascular outcomes with left atrial size: is volume superior to area or diameter? J Am Coll Cardiol 47(5):1018-1023
11. Rodevan O, Bjornerheim R, Ljosland M, Maehle J, Smith HJ, Ihlen $H$ (1999) Left atrial volumes assessed by three- and twodimensional echocardiography compared to MRI estimates. Int J Card Imaging 15(5):397-410

12. Keller AM, Gopal AS, King DL (2000) Left and right atrial volume by freehand three-dimensional echocardiography: in vivo validation using magnetic resonance imaging. Eur J Echocardiogr 1(1):55-65

13. Jenkins C, Bricknell K, Marwick TH (2005) Use of real-time three-dimensional echocardiography to measure left atrial volume: comparison with other echocardiographic techniques. J Am Soc Echocardiogr 18(9):991-997

14. Anwar AM, Soliman OI, Geleijnse ML, Nemes A, Vletter WB, ten Cate FJ (2008) Assessment of left atrial volume and function by real-time three-dimensional echocardiography. Int $\mathrm{J}$ Cardiol 123(2):155-161

15. Artang R, Migrino RQ, Harmann L, Bowers M, Woods TD (2009) Left atrial volume measurement with automated border detection by 3-dimensional echocardiography: comparison with Magnetic Resonance Imaging. Cardiovasc Ultrasound 7:16

16. Russo C, Hahn RT, Jin Z, Homma S, Sacco RL, Di Tullio MR (2010) Comparison of echocardiographic single-plane versus biplane method in the assessment of left atrial volume and validation by real time three-dimensional echocardiography. J Am Soc Echocardiogr 23(9):954-960

17. Miyasaka Y, Tsujimoto S, Maeba H, Yuasa F, Takehana K, Dote $\mathrm{K}$, Iwasaka T (2011) Left atrial volume by real-time threedimensional echocardiography: validation by 64 -slice multidetector computed tomography. J Am Soc Echocardiogr 24(6): 680-686

18. Rohner A, Brinkert M, Kawel N, Buechel RR, Leibundgut G, Grize L, Kuhne M, Bremerich J, Kaufmann BA, Zellweger MJ, Buser P, Osswald S, Handke M (2011) Functional assessment of the left atrium by real-time three-dimensional echocardiography using a novel dedicated analysis tool: initial validation studies in comparison with computed tomography. Eur $\mathbf{J}$ Echocardiogr 12(7):497-505

19. Lang RM, Bierig M, Devereux RB, Flachskampf FA, Foster E, Pellikka PA, Picard MH, Roman MJ, Seward J, Shanewise JS, Solomon SD, Spencer KT, Sutton MS, Stewart WJ (2005) Recommendations for chamber quantification: a report from the American Society of Echocardiography's Guidelines and Standards Committee and the Chamber Quantification Writing Group, developed in conjunction with the European Association of Echocardiography, a branch of the European Society of Cardiology. J Am Soc Echocardiogr 18(12):1440-1463

20. Aune E, Baekkevar M, Roislien J, Rodevand O, Otterstad JE (2009) Normal reference ranges for left and right atrial volume indexes and ejection fractions obtained with real-time threedimensional echocardiography. Eur J Echocardiogr 10(6):738744

21. Hauser TH, Peters DC, Wylie JV, Manning WJ (2008) Evaluating the left atrium by magnetic resonance imaging. Europace 10 Suppl 3:iii22-iii27

22. Cho GY, Jo SH, Kim MK, Kim HS, Park WJ, Choi YJ, Hong KS, Oh DJ, Rhim CY (2009) Left atrial dyssynchrony assessed by strain imaging in predicting future development of atrial fibrillation in patients with heart failure. Int J Cardiol 134(3):336-341

23. Marsan NA, Bleeker GB, Ypenburg C, Van Bommel RJ, Ghio S, Van de Veire NR, Delgado V, Holman ER, van der Wall EE, Schalij MJ, Bax JJ (2008) Real-time three-dimensional echocardiography as a novel approach to assess left ventricular and left atrium reverse remodeling and to predict response to cardiac resynchronization therapy. Heart Rhythm 5(9):1257-1264 